\title{
Uma Análise de Desempenho do Wi-Fi Direct para Comunicações Veículo-Pedestre
}

\author{
Thales T. Almeida ${ }^{1,2}$, José Geraldo R. Júnior ${ }^{1,2}$, \\ Miguel Elias M. Campista ${ }^{1}$ e Luís Henrique M. K. Costa ${ }^{1}$ \\ ${ }^{1}$ Universidade Federal do Rio de Janeiro (UFRJ) - PEE/COPPE/GTA \\ ${ }^{2}$ Centro Federal de Educação Tecnológica de Minas Gerais (CEFET-MG)
}

\begin{abstract}
Resumo. O alto custo dos dispositivos, associado à inexistência de um padrão universal, tem freado a implantação de VANETs baseadas no IEEE 802.11p, especialmente em países de baixa ou média renda. Neste sentido, sistemas baseados no Wi-Fi Direct têm se mostrado uma alternativa, tanto pelo seu potencial em integrar pedestres e ciclistas à VANET - não factível com o 802.11p devido à exigência de hardware compatível - quanto por permitir a adoção imediata destas redes baseado na alta penetração de smartphones. Com o objetivo de investigar a capacidade do Wi-Fi Direct em oferecer conectividade no ambiente veicular, este trabalho realiza uma análise de desempenho com base nos requisitos de uma aplicação de segurança. Em cenários com comunicação V2P, com e sem linha de visada, experimentos utilizando smartphones foram realizados a fim de avaliar o alcance da comunicação, a taxa de entrega e o tempo entre recepções de pacotes. O impacto de diferentes velocidades do veículo também foi avaliado. Os resultados indicam que, apesar das limitações, o Wi-Fi Direct tem potencial para servir como alternativa ao $802.11 \mathrm{p} \mathrm{em} \mathrm{aplicações} \mathrm{V2P.}$
\end{abstract}

\section{Introdução}

O número cada vez maior de veículos nas ruas, associado ao aumento constante no número de acidentes de trânsito, afeta importantes áreas como saúde pública e economia. Um relatório produzido pela OMS (Organização Mundial da Saúde) em 2018 aponta que, a cada ano, cerca de 1,35 milhão de pessoas morrem como resultado de lesões causadas por acidentes de trânsito [World Health Organization 2019]. Este problema é ainda mais grave em países de baixa e média renda. Mesmo concentrando $60 \%$ da frota terrestre, estes países respondem por mais de $90 \%$ das fatalidades. Para ilustrar, somente em 2015, mais de 38 mil pessoas perderam a vida em decorrência de acidentes de trânsito no Brasil.

Uma vez que aumentar a infraestrutura viária no mesmo ritmo que aumenta a frota de veículos é inviável, a pesquisa envolvendo VANETs (Vehicular Ad-hoc NETworks) tem se tornado cada vez mais atrativa. Em 1999, a americana FCC (Federal Communications Commission) alocou uma banda de $75 \mathrm{MHz}$ na faixa de 5,9 GHz para comunicações veiculares, o que permitiu o desenvolvimento, em 2010, do padrão IEEE 802.11p. Por meio de OBUs (On-Board Units), instaladas nos veículos, e RSUs (Road-Side Units), instaladas nas vias, é possível aumentar a segurança no trânsito através da troca de mensagens CAMs (Cooperative Awareness Messages) ou DENs (Decentralized Environmental Notifications), que permitem propagar o estado do ambiente e situações de emergência.

Apesar de ter sido projetado para VANETs, o 802.11p tem demonstrado grande dificuldade de implantação no mundo real [Miucic and Bai 2011]. Isto se deve, principalmente, ao alto custo dos dispositivos compatíveis, à falta de um padrão de comunicação 
universal (há diferenças entre o padrão europeu, japonês e americano), bem como à falta de aplicações que atraiam o interesse dos condutores. Como o $802.11 \mathrm{p}$ requer que os veículos sejam equipados com hardware dedicado, um grande investimento inicial necessário [Balasundram et al. 2016]. Este cenário dificulta a penetração em países de baixa e média renda, e leva à busca por métodos alternativos de comunicação entre veículos.

De acordo com [Statista 2019], 44,9\% da população mundial terá um smartphone em 2020. Dado este poder de penetração, tecnologias de rede embarcadas nestes dispositivos são vistas como uma alternativa ao 802.11 p, capazes de permitir a adoção imediata de VANETs e a integração pedestres e ciclistas ao contexto veicular. Com estas tecnologias, é possível compartilhar dados coletados por sensores (como posição e velocidade), e prever, por exemplo, a colisão entre veículos portando um smartphone (como uma OBU no 802.11p). São muitas as tecnologias que permitem tal compartilhamento, como NFC (Near Field Communication), BLE (Bluetooth Low Energy), redes 4G/5G e Wi-Fi Direct. Introduzido em 2010 pela Wi-Fi Alliance ${ }^{1}$, o Wi-Fi Direct possui o melhor benefício.

Enquanto o NFC não é cogitado dado o seu alcance de $10 \mathrm{~cm}$ [Jeong et al. 2016], e o BLE é capaz de enviar dados a até $100 \mathrm{~m}$ [Frank et al. 2014], o Wi-Fi Direct possui alcance teórico de $200 \mathrm{~m}$ [Khan et al. 2017]. Embora inferior ao 802.11p (1.000 m), o alcance de $200 \mathrm{~m}$ do Wi-Fi Direct é capaz de suportar algumas aplicações de segurança, como alerta de ponto cego e de mudança de faixa, que atuam dentro de $100 \mathrm{~m}$ de cobertura [Jeong et al. 2019]. Além disso, enquanto o BLE suporta 1 Mbps [Touati et al. 2013], no Wi-Fi Direct é possível transmitir dados à taxa de $250 \mathrm{Mbps}$, nove vezes superior à taxa máxima do 802.11p (27 Mbps). Ademais, o atraso fim-a-fim no Wi-Fi Direct é inferior ao do 4G [Jeong et al. 2019], que não atende aos requisitos de aplicações de segurança [Park et al. 2014]. Apesar do potencial, não há estimativas de quando o 5G entrará em operação. Já o Wi-Fi Direct está presente na maioria dos smartphones. Isto possibilita a imediata adoção das VANETs, algo que não se projeta com o 802.11p e 5G. Por fim, dado o tamanho mínimo (50 B) de uma BSM (Basic Safety Message) e frequência de transmissão $(10 \mathrm{~Hz})$ de aplicações de segurança, uma carga de 1,72 MB/hora seria gerada. Em redes celulares, o custo para o usuário e a sobrecarga da rede devem ser considerados.

A principal desvantagem do Wi-Fi Direct diz respeito ao longo tempo para estabelecimento da conexão, compreendida de duas etapas [Khan et al. 2017]: (1) descoberta de pares; e (2) formação do grupo. Na descoberta de pares, o objetivo é detectar a presença de outros nós. Isto é feito em duas fases: Scan e Find. Durante o Scan, o nó sensoria todos os canais em busca de outros nós ou grupos. Após esta fase, se inicia a fase de Find, que é dividida entre Search e Listen. Durante o Search, são transmitidos quadros de requisição nos canais 1,6 e 11. Já durante o Listen, o nó seleciona um destes canais à espera destes mesmos quadros. Uma conexão é realizada se dois nós sincronizam em um mesmo canal. Após a descoberta, os nós podem formar um grupo equivalente a um BSS (Basic Service Set). No Wi-Fi Direct, três grupos podem ser formados: (1) Standard, onde há uma negociação entre os nós sobre qual deles será o GO (Group Owner), semelhante ao ponto de acesso; (2) Autonomous, onde um nó se anuncia como GO através de beacons; e (3) Persistent, que permite reativar um grupo que já existiu. Antes de trocar de dados, uma conexão segura precisa ser estabelecida através do WPS (Wi-Fi Protected Setup).

\footnotetext{
${ }^{1}$ https://www.wi-fi.org/wi-fi-direct
} 
Conforme [Camps-Mur et al. 2013], o tempo para estabelecer a conexão pode ser de $15 \mathrm{~s}$. Muitos trabalhos visam reduzir este tempo [Zhang et al. 2014, Chaki et al. 2015, Sun et al. 2016]. Entretanto, para o uso em VANETs, é necessário avaliar, a priori, a capacidade da rede de disseminar dados a partir da avaliação de diferentes métricas. Como o Wi-Fi Direct é projetado para ambientes estacionários [Jeong et al. 2019], é preciso analisar seu desempenho sob condições de mobilidade, investigando, por exemplo, se a velocidade dos veículos impacta as transmissões devido ao deslocamento Doppler. Caso esta análise indique que o Wi-Fi Direct não obtém bom desempenho em VANETs, qualquer esforço para reduzir o tempo de estabelecimento da conexão se mostrará dispensável.

Com base nesta premissa, este trabalho investiga a capacidade do Wi-Fi Direct em atender aos requisitos de operação de uma aplicação de segurança no ambiente veicular. Baseado na troca periódica de BSMs entre um veículo e um pedestre (V2P - Vehicle-ToPedestrian), são analisados os resultados de medições reais usando smartphones comerciais. Em cenários com e sem linha de visada (LoS - Line-of-Sight e NLoS - Non-Line-ofSight), são avaliados o alcance máximo da comunicação, a PDR (Packet Delivery Rate) e o PIR (Packet Inter-Reception time). O impacto de diferentes velocidades empregadas pelo veículo também foi investigado. De acordo com nosso conhecimento, este é o primeiro estudo que analisa o uso do Wi-Fi Direct em VANETs por meio de experimentos de campo em cenários com condições de LoS e NLoS, e altas velocidades relativas.

O restante deste artigo possui a seguinte estrutura: a Seção 2 apresenta os trabalhos relacionados. Na Seção 3 são apresentados os cenários dos experimentos reais, além dos detalhes de implementação e configuração. A Seção 4 disserta sobre os resultados dos experimentos. Por fim, a Seção 5 conclui o artigo e apresenta os trabalhos futuros.

\section{Trabalhos Relacionados}

Como mencionado na Seção 1, muitos trabalhos envolvendo o Wi-Fi Direct são propostos com o objetivo de reduzir o tempo para estabelecimento da conexão. Em menor número, alguns possuem como foco a análise do comportamento do Wi-Fi Direct em VANETs.

Em [Camps-Mur et al. 2013], os autores apresentam a primeira análise de desempenho do Wi-Fi Direct por meio de experimentos reais e simulações no NS-3. O objetivo é analisar o atraso na formação dos três grupos do Wi-Fi Direct e comparar estes resultados com os obtidos no Wi-Fi tradicional. Os resultados indicam que o atraso para descoberta no modo Standard e Persistent são similares, e que o modo Autonomous obtém o menor atraso. Quanto ao atraso para formação do grupo, o modo Persistent obteve o melhor resultado. O atraso de formação dos grupos Standard, Autonomous e Persistent foram, respectivamente, $<9 \mathrm{~s}, 5 \mathrm{~s}$ e $8 \mathrm{~s}$. Por não considerar interferência, as simulações não refletiram os experimentos reais. Além disso, o NS-3 não possui módulos do Wi-Fi Direct. Os autores não mencionam nenhuma adaptação deste módulo.

Os autores em [Su et al. 2012] propõem um dos primeiros trabalhos a considerar o Wi-Fi Direct em VANETs. Entretanto, devido ao atraso gerado pelo aceite do usuário à conexão, os autores decidiram por implementar o modo $A d$ hoc nos dispositivos. Ao contrário do Wi-Fi Direct, por segurança o modo Ad hoc não é suportado nos smartphones, a menos que seja habilitado o root. Por meio de experimentos reais, os autores avaliaram o alcance máximo da comunicação e o atraso de transmissão. $\mathrm{O}$ alcance foi medido com os smartphones dentro e fora dos veículos, com e sem movimento. Os resultados 
indicam que o alcance, com os dispositivos fora do veículo, foi de $200 \mathrm{~m}$. Dentro dos veículos imóveis, o alcance caiu para $100 \mathrm{~m}$ com as janelas abertas e $50 \mathrm{~m}$ com as janelas fechadas. O movimento dos veículos ( $45 \mathrm{~km} / \mathrm{h}$ e $90 \mathrm{~km} / \mathrm{h}$ ) não impactou os resultados. Porém, nos testes de velocidade não foram usados os mesmos veículos, o que pode levar a diferenças nos resultados. Por fim, o atraso médio de transmissão foi de cerca de $30 \mathrm{~ms}$.

Em [Jeong et al. 2016], com foco na redução do atraso para descoberta, os autores combinam o Wi-Fi Direct com redes 4G visando a operação de aplicações de segurança em VANETs. Um servidor é responsável por instruir, através do 4G, quando os dispositivos devem estabelecer conexões via Wi-Fi Direct. Cada dispositivo envia ao servidor, através da rede $4 \mathrm{G}$, sua posição geográfica e velocidade. O servidor monitora a distância entre os nós e, sempre que a mesma for inferior a $200 \mathrm{~m}$, envia uma solicitação para que os nós formem um grupo de comunicação. Após esta etapa, e a consequente escolha do GO pelo servidor, a troca de dados pode ser realizada via Wi-Fi Direct. A avaliação foi realizada com veículos a $20 \mathrm{~km} / \mathrm{h}$ e $30 \mathrm{~km} / \mathrm{h}$, e contendo smartphones em seu interior. Os resultados indicam que o tempo de descoberta caiu de $1,5 \mathrm{~s}$ para $100 \mathrm{~ms}$. No cenário que demonstra a desconexão do GO, o tempo para descoberta caiu de $3 \mathrm{~s}$ para $200 \mathrm{~ms}$. Os autores, porém, não consideraram o atraso com o provisionamento WPS, que permaneceu alto em todos os cenários. Além disso, nenhuma análise da PDR com relação à velocidade do veículo, impacto de condições NLoS, entre outros, foi realizada.

Em [Manamperi et al. 2018], visando reduzir o atraso de transmissão e impulsionar o Wi-Fi Direct em VANETs, os autores propõem uma transmissão broadcast entre o GO e os clientes, substituindo o modelo P2P (Peer-To-Peer). Na proposta, o GO recebe os quadros enviados pelos clientes e agrega em um quadro único, enviado a todos os clientes. A avaliação foi realizada no simulador INET, onde o IEEE $802.11 \mathrm{~b}$ foi adaptado para simular o Wi-Fi Direct, considerando a taxa de $6 \mathrm{Mbps}$, frequência de 2,4 GHz e mensagens transmitidas a $5 \mathrm{~Hz}$. O alcance foi definido em $250 \mathrm{~m}$. A avaliação contou com veículos a $80 \mathrm{~km} / \mathrm{h}$ e $120 \mathrm{~km} / \mathrm{h}$. Os resultados indicam que, sem ACKs e retransmissões, o atraso médio total foi reduzido em comparação à comunicação tradicional, apesar do aumento do número de clientes levar a uma redução da taxa de recepção. Além de alguns parâmetros da simulação serem pouco realísticos (como o expoente de perda do modelo de propagação), o uso do 802.11 b pode não refletir o funcionamento do Wi-Fi Direct.

Usando o módulo do IEEE 802.11a, em [Balasundram et al. 2016] os autores avaliam o Wi-Fi Direct por meio de simulações no o NS-3, onde a vazão, o atraso fim-a-fim e a PDR são comparadas com as obtidas no 802.11p. Dois cenários de transmissão são considerados: (1) um salto, realizada por dois veículos com destino a uma RSU; (2) múltiplos saltos, entre veículos de diferentes clusters. Com relação à PDR, os resultados indicam, no Wi-Fi Direct, um aumento da perda com o aumento da distância, acentuada a partir de $150 \mathrm{~m}$. Apesar disso, é possível ter comunicação até $300 \mathrm{~m}$. Este resultado conflita com o alcance máximo teórico do Wi-Fi Direct $(200 \mathrm{~m})$, e pode estar associado ao uso do 802.11a. Com relação à vazão, o mesmo comportamento de queda foi observado. Na transmissão por múltiplos saltos, a média do atraso fim-a-fim foi superior no Wi-Fi Direct em comparação ao 802.11p, ao passo que a média da PDR foi inferior. Por fim, a velocidade do veículo não influencia a PDR, já que os modelos de propagação não consideram a mobilidade. Além disso, o atraso para formação de grupos foi ignorado.

Em [Shahin and Younis 2015], os autores propõem incorporar uma mensagem de 
alerta no quadro do mecanismo opcional de descoberta de serviços do Wi-Fi Direct. Na proposta, não é necessário estar em um grupo para transmitir dados. Nós interessados em receber alertas enviam um quadro de requisição ao nó com alertas armazenados, que os transmite após receber o quadro. A proposta também permite reencaminhar alertas, controlando a inundação por meio de um TTL (Time-To-Live). Implementado em dispositivos reais, os alertas são baseados na posição geográfica obtida a partir da interação do usuário com um mapa. Testes de validação foram realizados, enquanto que a certificação do protocolo em condições reais não foi descrita pelos autores. Apesar de evitar o atraso na formação de grupos, o modelo publish/subscribe, onde alertas só são enviados após requisição, não é adequado para aplicações de segurança, já que a falta de envio periódico das BSMs prejudica a consciência situacional e aumenta o risco de colisões.

Com foco em avaliar o comportamento do Wi-Fi Direct em suportar aplicações de segurança, em [Won et al. 2018] os autores propõem um sistema para monitorar o risco de colisões envolvendo veículos e pedestres. Além de propagar alertas, o sistema estabelece mecanismos para aumento da precisão do GPS, economia de energia e detecção de sinais de distração do pedestre baseado no uso do smartphone. Após detectar uma visualização de tela enquanto o pedestre caminha junto à faixa, o sistema cria um grupo Autonomous entre o pedestre (GO) e os veículos. Os dispositivos trocam mensagens contendo o tempo para que o pedestre e o veículo alcancem o cruzamento. Com base na recepção da mensagem pelo pedestre, o algoritmo calcula a probabilidade de colisão, e um alerta é enviado. $\mathrm{Na}$ avaliação, os autores desconsideram o tempo para estabelecimento da conexão. Além disso, para permitir a comunicação NxN no Wi-Fi Direct, foi proposta uma estratégia baseada na inclusão de um novo pedestre a um grupo já criado, com a comunicação do novo membro intermediada pelo GO. Os autores analisaram o alcance da comunicação (LoS) com base na PDR e o atraso fim-a-fim com base no RTT (Round-Trip-Time). Os resultados indicam que a PDR é $>80 \%$ em distâncias $<70 \mathrm{~m}$, e que o RTT ficou entre $100 \mathrm{~ms}$ e 200 ms. Quanto menor é a velocidade do veículo, maior é a chance de evitar uma colisão. Por fim, os resultados apontam que, no geral, a PDR independe da velocidade do veículo, apesar da ocorrência de uma pequena queda em velocidades mais altas.

Como pode ser visto, muitos trabalhos envolvendo o Wi-Fi Direct são baseados no uso de simuladores, que podem não possuir módulos de comunicação compatíveis com o Wi-Fi Direct. Como [Won et al. 2018], este trabalho analisa se o Wi-Fi Direct atende aos requisitos de uma aplicação de segurança em VANETs usando dispositivos reais. Porém, ao contrário de [Won et al. 2018] - onde a velocidade máxima do veículo foi $30 \mathrm{~km} / \mathrm{h}$ neste trabalho velocidades de até $100 \mathrm{~km} / \mathrm{h}$ são empregadas. Além disso, nos experimentos de [Won et al. 2018], o atraso para estabelecimento da conexão foi desconsiderado. Já no presente trabalho, a conexão é feita durante os experimentos de mobilidade, de forma a investigar seu impacto efetivo na comunicação. Uma importante contribuição em relação a [Won et al. 2018] é que a presente análise é feita em cenários com e sem condições de LoS, aproximando os experimentos das situações encontradas no mundo real. Por fim, além do alcance máximo da comunicação e da PDR, investiga-se também o PIR, dada sua importância como métrica de desempenho para aplicações de segurança.

\section{Experimentos com o Wi-Fi Direct}

A avaliação é composta por um pedestre portando um smartphone que atua como cliente do grupo, bem como por um veículo contendo um smartphone que atua como GO. 


\subsection{Cenários Experimentais}

Os cenários de avaliação do Wi-Fi Direct têm como base uma via sinalizada (Figura 1(a)), onde pedestres podem realizar a travessia na faixa enquanto veículos aguardam por autorização semafórica. No mundo real, a travessia de pedestres ou veículos quando não há autorização semafórica é uma situação comum, que coloca em risco a vida de pedestres e condutores. Tal situação é agravada com a presença de obstáculos que inibem a propagação do sinal de rádio (como um ônibus parado coletando passageiros). Neste caso, além da obstrução do sinal, há também o bloqueio do campo visual do pedestre, que não percebe a chegada de veículos ao tentar fazer a travessia sem a devida autorização semafórica. Neste sentido, três cenários foram definidos, ilustrados na Figura 1(b).

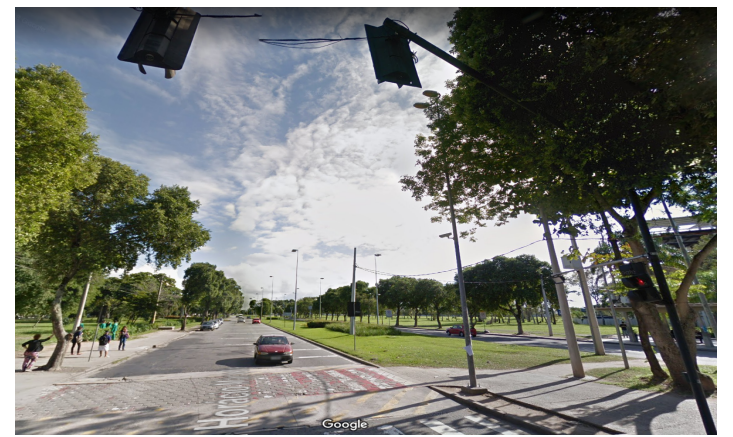

(a) Exemplo de via sinalizada (Campus UFRJ).

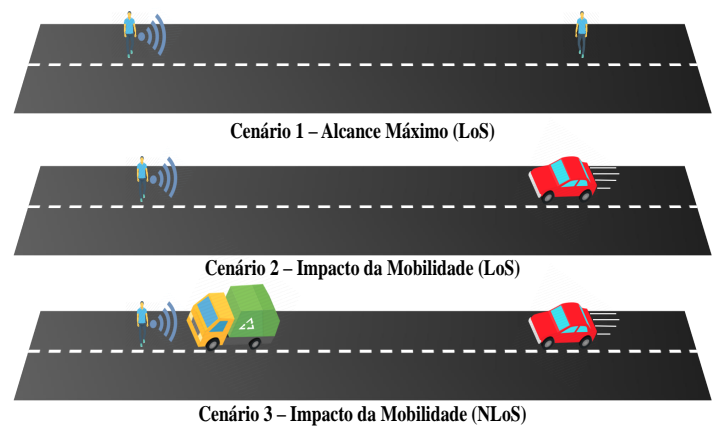

(b) Situações avaliadas no cenário experimental.

Figura 1. Exemplo de um cenário real e situações avaliadas.

Cenário 1 - Alcance Máximo (LoS): analisa se o alcance máximo obtido pelo Wi-Fi Direct no mundo real corresponde ao alcance máximo teórico de $200 \mathrm{~m}$. O objetivo, além de analisar o alcance real, é servir como referência para cenários com mobilidade. Neste cenário, após o estabelecimento da conexão, dois pedestres estáticos portando smartphones se comunicam por meio de BSMs. A avaliação é baseada na transmissão de um conjunto de $500 \mathrm{BSMs}$, de $10 \mathrm{~m}$ em $10 \mathrm{~m}$, até que os dispositivos estejam a $200 \mathrm{~m}$ de distância. O alcance máximo da comunicação é definido como a distância máxima no qual é possível receber, no mínimo, 80\% das BSMs transmitidas [Bai et al. 2010].

Cenário 2 - Impacto da Mobilidade (LoS): mede o impacto da mobilidade no desempenho do Wi-Fi Direct. Enquanto o pedestre se mantém estático e transmitindo BSMs continuamente, o veículo se locomove em direção ao pedestre com velocidades de $20 \mathrm{~km} / \mathrm{h}$, $60 \mathrm{~km} / \mathrm{h}$ ou $100 \mathrm{~km} / \mathrm{h}$. O veículo inicia sua trajetória a $500 \mathrm{~m}$ do pedestre, distância suficientemente grande para entrar no alcance de comunicação do pedestre já tendo alcançado a velocidade desejada. O objetivo é investigar, por exemplo, se o deslocamento Doppler causado pela mobilidade do nó é capaz de impactar a comunicação com base na PDR e no PIR. Ao contrário do cenário 1, todas as transmissões feitas durante a rota do veículo são contabilizadas, e o cálculo da PDR e do PIR é feito a cada $20 \mathrm{~m}$.

Cenário 3 - Impacto da Mobilidade (NLoS): similar ao cenário 2, com o adendo de inserir um veículo de médio porte (sem Wi-Fi Direct) entre o pedestre e o veículo atuando como GO. O objetivo é analisar o impacto causado pela obstrução do sinal de rádio, causada por condições NLoS na comunicação em um ambiente mais realista. No mundo real, não existem garantias de linha de visada entre o transmissor e o receptor. $\mathrm{O}$ sinal 
pode ser afetado por árvores, edificações ou outros veículos presentes na via. Portanto, é preciso avaliar se as BSMs serão capazes de alertar o veículo em movimento sobre a travessia indevida do pedestre, que não possui informação visual devido ao obstáculo.

A análise de desempenho do Wi-Fi Direct tem como base a PDR e o PIR. Enquanto a PDR indica o percentual de BSMs recebidas em função do total das BSMs transmitidas, o PIR demonstra o intervalo de tempo entre duas recepções bem sucedidas. Esta métrica reflete o nível de consciência situacional: através das BSMs, um veículo obtém a posição, velocidade e estado dos demais veículos e pedestres na via. Quanto maior é a consciência situacional, menor é o tempo de reação sob uma condição adversa. Das análises de desempenho na literatura, boa parte investiga apenas a PDR. Entretanto, dado que o objetivo é analisar o Wi-Fi Direct para a operação de uma aplicação de segurança, a análise do PIR é essencial para indicar uma possível ausência de comunicação por longos períodos. A Tabela 1 resume os três cenários de avaliação.

Tabela 1. Características dos Cenários Experimentais.

\begin{tabular}{|c|c|c|c|c|c|}
\hline Cenário & Verificação & Condição & Vel. Pedestre & Vel. Veículo & Taxa PHY \\
\hline 1 & Alcance Máximo & $\mathrm{LoS}$ & $0 \mathrm{~km} / \mathrm{h}$ & $0 \mathrm{~km} / \mathrm{h}$ & $250 \mathrm{Mbps}$ \\
\hline 2 & Impacto da Mobilidade & $\mathrm{LoS}$ & $0 \mathrm{~km} / \mathrm{h}$ & $20-60-100 \mathrm{~km} / \mathrm{h}$ & $250 \mathrm{Mbps}$ \\
\hline 3 & Impacto da Mobilidade & $\mathrm{NLoS}$ & $0 \mathrm{~km} / \mathrm{h}$ & $20-60-100 \mathrm{~km} / \mathrm{h}$ & $250 \mathrm{Mbps}$ \\
\hline
\end{tabular}

\subsection{Implementação e Local dos Experimentos}

Os experimentos foram realizados utilizando dois smartphones comerciais: (1) Xiaomi MI A2, com processador Octa-Core de $2.0 \mathrm{GHz}$ e $4 \mathrm{~GB}$ de RAM, atuando como GO; e (2) Asus Zenfone Live L1, com processador Octa-Core de $1.4 \mathrm{GHz}$ e $2 \mathrm{~GB}$ de RAM, atuando como cliente do grupo. As versões do Android dos dispositivos são a 9.0 One Pie (API 28) para o Xiaomi, e a 8.0 Oreo (API 26) para o Asus. Desde a versão 4.0 (Ice Cream Sandwich), de 2011, o Wi-Fi Direct encontra-se disponível no Android.

Como o intuito é analisar o desempenho do Wi-Fi Direct sob aspectos da rede, o atraso baseado na interação do usuário com a tela - para seleção do nó e estabelecimento da conexão - foi ignorado em todos os cenários. Isto foi realizado invocando o método de conexão do Wi-Fi Direct dentro do método PeerListListener ${ }^{2}$ - responsável por mostrar na tela a lista de pares disponíveis após a conclusão de etapa de descoberta - e passando de maneira explícita o ID do dispositivo atuando como GO. O mesmo se deu em relação à transmissão de BSMs: tão logo o grupo é formado, o cliente transmite BSMs via socket UDP ao GO. Nenhuma interação com a aplicação por parte do usuário é necessária.

A comunicação entre o pedestre e o veículo consiste na transmissão de BSMs representando uma mensagem CAM, responsável por propagar o estado do ambiente. Cada BSM possui as coordenadas geográficas do nó (latitude e longitude), obtidas por meio do GPS com taxa de atualização de $1 \mathrm{~Hz}$, sua velocidade atual, sentido de direção, e timestamp da informação. As BSMs são transmitidas a cada $100 \mathrm{~ms}$, atendendo ao requisito de frequência mínima requerido por aplicações de segurança em VANETs [Consortium et al. 2005]. Cada BSM possui em torno de $80 \mathrm{~B}$ de tamanho. As janelas do veículo permaneceram fechadas durante toda a comunicação com o pedestre.

\footnotetext{
${ }^{2}$ https://developer.android.com/reference/android/net/wifi/p2p/WifiP2pManager.PeerListListener
} 
Os experimentos foram realizados em um aeroporto desativado da cidade de Leopoldina - MG. Contando com uma pista de 1,2 km de extensão e estando em uma área rural, o local demonstrou ser o ideal para a realização dos experimentos: não recebe interferência de redes celulares ou Wi-Fi e, por estar em uma área predominantemente aberta, não possui edificações que prejudicariam a avaliação do cenário com LoS. A Figura 2(a) apresenta a visão aérea do local, enquanto as Figuras 2(b), 2(c), 2(d) e 2(e) apresentam os dispositivos utilizados e a avaliação do Wi-Fi Direct em progresso, respectivamente.

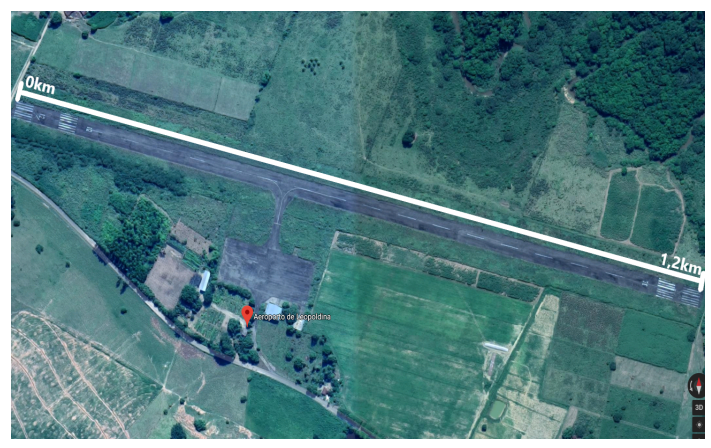

(a) Local dos experimentos.

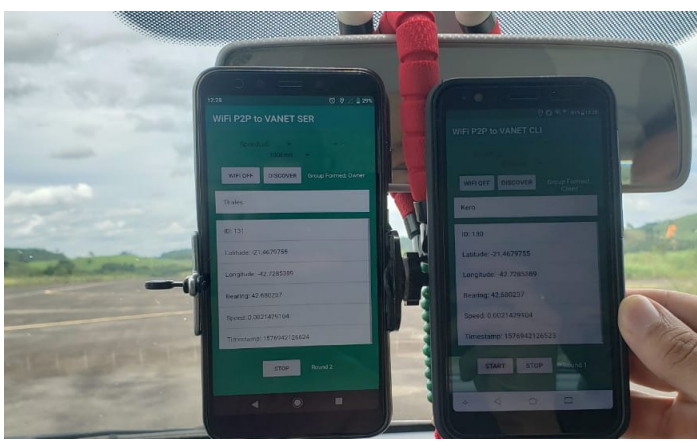

(b) Dispositivos utilizados.

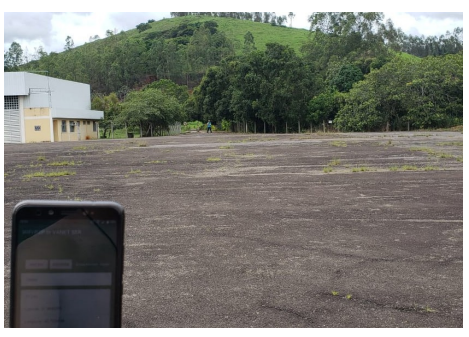

(c) Alcance máximo.

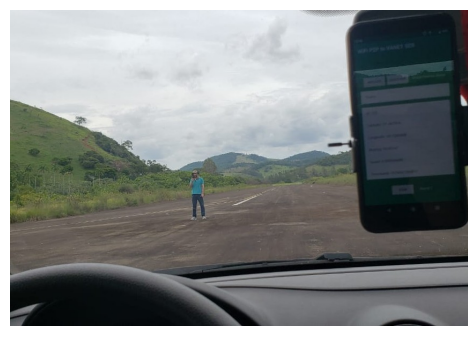

(d) Mobilidade com LoS.

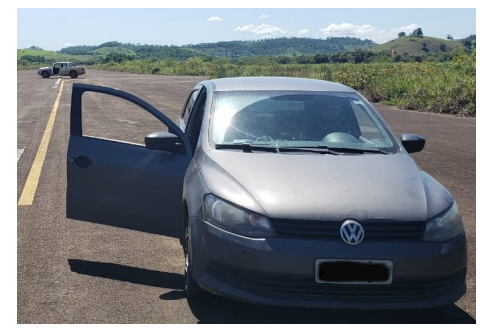

(e) Mobilidade com NLoS.

Figura 2. Local dos testes, dispositivos utilizados e avaliação em curso.

\section{Resultados}

Esta seção apresenta os resultados da avaliação prática do Wi-Fi Direct. Para cada cenário definido na Seção 3, 10 rodadas de experimentos foram executadas. A mediana dos resultados é apresentada contendo barras verticais de erro, que correspondem ao desvio absoluto da mediana. A escolha pela mediana se deu pela sua robustez em tratar outliers.

\subsection{Alcance Máximo da Communicação}

Antes de considerar o Wi-Fi Direct no ambiente veicular, é preciso analisar se os dispositivos conseguirão se comunicar a uma distância que atenda aos requisitos da aplicação. O objetivo deste cenário é investigar se o alcance máximo teórico de $200 \mathrm{~m}$ do Wi-Fi Direct pode ser obtido na prática. Além disso, os resultados deste cenário servem como referência para analisar o impacto causado pela mobilidade e pelas condições NLoS na comunicação. As Figuras 3(a) e 3(b) apresentam o alcance máximo real do Wi-Fi Direct.

Iniciando a avaliação a partir de $50 \mathrm{~m}$, a até $100 \mathrm{~m}$ de distância a PDR obtida foi próxima de $100 \%$. Apesar de $\geq 60 \%$ a até $150 \mathrm{~m}$, é notória a queda da PDR a partir de $110 \mathrm{~m}$, mais evidente em $200 \mathrm{~m}$. Portanto, apesar de ainda ser possível transmitir BSMs a 200 m de distância, o alcance máximo real do Wi-Fi Direct no mundo real - considerando 


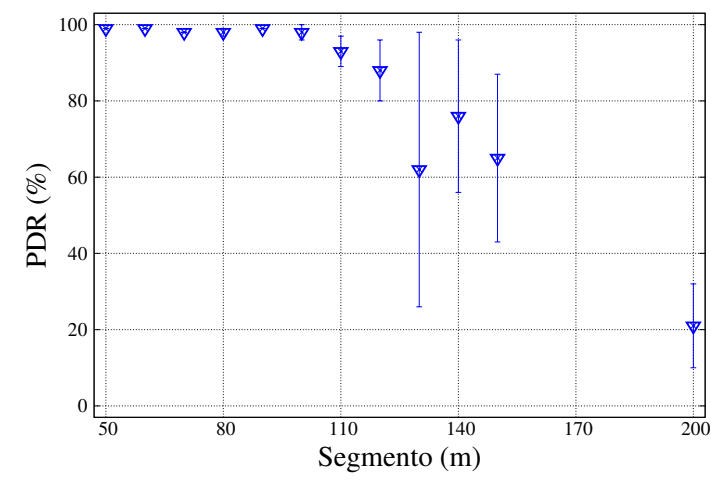

(a) PDR obtida no cenário 1 .

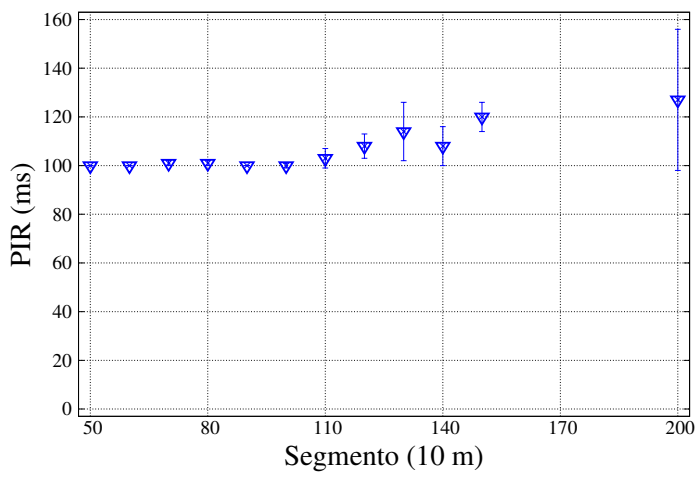

(b) PIR obtido no cenário 1.

Figura 3. Alcance máximo da comunicação.

a PDR $\geq 80 \%$ - foi de 120 m. Por sinal, a PDR de $20 \%$ em 200 m pode não garantir a plena operação de uma aplicação de segurança neste ponto da via. Com relação ao PIR, é possível observar uma correlação negativa com a PDR, especialmente a partir de $110 \mathrm{~m}$. Quanto menor é a PDR, maior é o PIR. Dado que a potência de transmissão é fixa, quanto maior é o alcance da comunicação, mais forte é a atenuação do sinal de rádio, impactando a PDR e o PIR. Isto pode indicar um alerta para a operação de aplicações que dependam de comunicação a longas distâncias.

\subsection{Impacto da Mobilidade na Comunicação (LoS)}

O segundo conjunto de experimentos investiga o impacto da mobilidade do veículo $20 \mathrm{~km} / \mathrm{h}, 60 \mathrm{~km} / \mathrm{h}$ e $100 \mathrm{~km} / \mathrm{h}$ - na comunicação realizada em condições de linha de visada (LoS). A PDR e o PIR são calculados a cada segmento de $20 \mathrm{~m}$, conforme o veículo se aproxima da posição do pedestre. As Figuras 4(a) e 4(b) apresentam os resultados obtidos para este cenário. Em todas as figuras, o eixo das abscissas é apresentado de forma decrescente, representando a aproximação do veículo em direção ao pedestre.

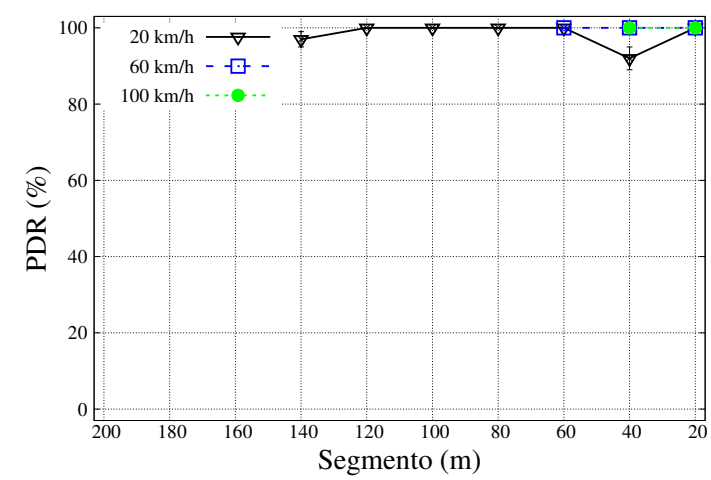

(a) PDR obtida no cenário 2.

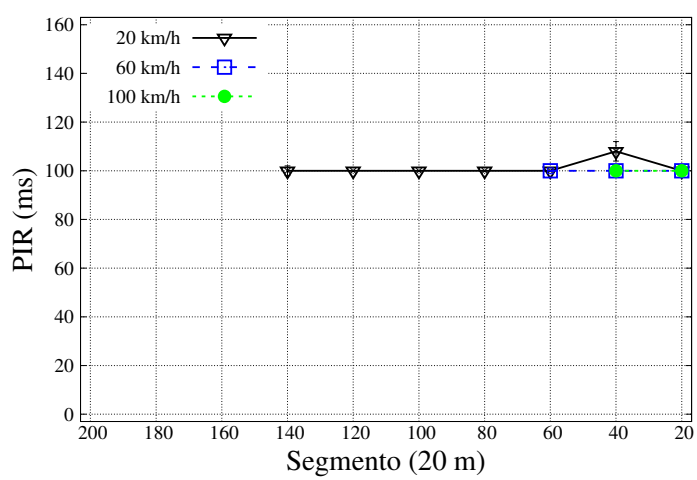

(b) PIR obtido no cenário 2.

Figura 4. Impacto da mobilidade em condições com LoS.

Os resultados da PDR demonstram o impacto do longo atraso para estabelecimento da conexão no Wi-Fi Direct em um cenário com mobilidade. Como demonstrado na Seção 4.1, no cenário estático é possível receber BSMs em $200 \mathrm{~m}$. Já no presente 
cenário, a distância máxima na qual o pedestre (cliente) conclui o estabelecimento da conexão com o veículo $(\mathrm{GO})$ - estando apto a transmitir BSMs - é de aproximadamente $140 \mathrm{~m}$, enquanto o veículo está a $20 \mathrm{~km} / \mathrm{h}$. A partir desta distância, a PDR a $20 \mathrm{~km} / \mathrm{h}$ foi $\geq$ $90 \%$. Já o estabelecimento da conexão - e transmissão de BSMs - a $60 \mathrm{~km} / \mathrm{h}$ e $100 \mathrm{~km} / \mathrm{h}$ se deu apenas nos últimos $60 \mathrm{~m}$ e $40 \mathrm{~m}$, respectivamente. Nestes casos, a PDR foi $100 \%$.

É possível calcular a chance de colisão entre veículo e pedestre com base na soma do tempo de reação do motorista após receber o alerta e do tempo para parar o veículo após o acionamento dos freios, comparando o resultado com o tempo para o veículo atingir o pedestre. Tais parâmetros são afetados: (1) pela velocidade do veículo e cognição do motorista; e (2) pela condição de pneus, freios e estrada. A distância de parada do veículo após a recepção da BSM pode ser calculada com base na seguinte equação:

$$
D_{\text {total }}=d_{\text {reacao }}+d_{\text {frenagem }}=v_{\text {veiculo }} \cdot t_{\text {reacao }}+\frac{v_{\text {veiculo }}^{2}}{2 \mu g}
$$

onde $d_{\text {reacao }}$ é a distância percorrida durante a reação do motorista, e $d_{\text {frenagem }}$ é a distância percorrida após acionar os freios, com base na velocidade $\left(v_{\text {veiculo }}\right)$, coeficiente de fricção $(\mu)$, aceleração da gravidade $(g)$ e tempo de reação do motorista $\left(t_{\text {reacao }}\right)$.

Em [Won et al. 2018], os autores também calculam as chances de colisão, porém agregando o RTT e alguns outros parâmetros em $t_{\text {frenagem }}$. Neste trabalho, para cálculo da chance de colisão, considerou-se, no pior caso, um atraso equivalente ao RTT de $100 \mathrm{~ms}$ (média do PIR), $t_{\text {reacao }}$ como $1 \mathrm{~s}$ [Renda et al. 2016], $g$ de $9,81 \mathrm{~m} / \mathrm{s}^{2}$, e dado que as medições foram realizadas em boas condições climáticas, $\mu$ de 0,8 (compatível com o asfalto seco) [Wong 2008]. Considerando $v_{\text {veiculo }}$ como $20 \mathrm{~km} / \mathrm{h}, 60 \mathrm{~km} / \mathrm{h}$ e $100 \mathrm{~km} / \mathrm{h}$, uma distância aproximada de $8 \mathrm{~m}, 36 \mathrm{~m}$ e $80 \mathrm{~m}$ seria percorrida, respectivamente, até que o veículo parasse por completo. De acordo com a Figura 4(a), apenas a $100 \mathrm{~km} / \mathrm{h}$ pode-se não ser possível evitar a colisão. Porém, com exceção das vias de trânsito rápido, a velocidade máxima permitida nas vias urbanas no Brasil é de $60 \mathrm{~km} / \mathrm{h}$. Neste caso, ao menos em condições de LoS, o Wi-Fi Direct atende satisfatoriamente a comunicação V2P.

Com relação ao PIR, é possível perceber uma correlação com a PDR obtida a $20 \mathrm{~km} / \mathrm{h}$ no segmento entre 20 e $40 \mathrm{~m}$ de distância. Nos casos onde a PDR foi próxima ou igual a 100\%, o PIR foi similar à taxa de BSMs (100 ms). Este resultado é importante pois demonstra que, estabelecida a conexão, o Wi-Fi Direct foi capaz de atender ao requisito de frequência de recepção de aplicações de segurança [Consortium et al. 2005]. Além disso, dado que o PIR máximo foi $110 \mathrm{~ms}$, nenhum blackout de consciência situacional (intervalo $\geq 1$ s no qual nenhuma BSM é recebida [Renda et al. 2016]) ocorreu durante os experimentos. Isto indica que em nenhum momento o veículo navegou às cegas pela via, sem estar ciente do estado do pedestre em tempo real.

Por fim, a Tabela 2 mostra a PDR e o PIR global, com base nas transmissões e recepções realizadas durante todo o percurso do veículo, sem dividir os resultados por segmentos. Dada a relação entre o aumento da velocidade do nó e o deslocamento Doppler da frequência - que supostamente causa um aumento da BER (Bit Error Rate) - é importante verificar se o emprego de altas velocidades (como $100 \mathrm{~km} / \mathrm{h}$ ) leva a uma piora da PDR e do PIR. Independente da velocidade, observa-se que a PDR é superior a $90 \%$ e o PIR é similar à taxa de BSMs, o que indica uma tolerância da camada física do Wi-Fi Direct à interferência intersimbólica gerada pelo deslocamento Doppler. 
Tabela 2. PDR e PIR global por velocidade (LoS).

\begin{tabular}{|c|c|c|}
\hline Velocidade (km/h) & PDR (\%) & PIR (ms) \\
\hline 20 & 97,55 & 102,99 \\
\hline 60 & 100 & 100,48 \\
\hline 100 & 100 & 100,56 \\
\hline
\end{tabular}

\subsection{Impacto da Mobilidade na Comunicação (NLoS)}

O último conjunto de experimentos investiga o comportamento da comunicação em um ambiente com mobilidade e sem linha de visada (NLoS). Neste cenário, o veículo se locomove a $20 \mathrm{~km} / \mathrm{h}, 60 \mathrm{~km} / \mathrm{h}$ e $100 \mathrm{~km} / \mathrm{h}$ em direção ao pedestre, que desta vez está posicionado atrás de um veículo de médio porte estático (caminhonete $4 \times 4$ ). Novamente, PDR e PIR são calculados a cada $20 \mathrm{~m}$. As Figuras 5(a) e 5(b) apresentam os resultados obtidos.

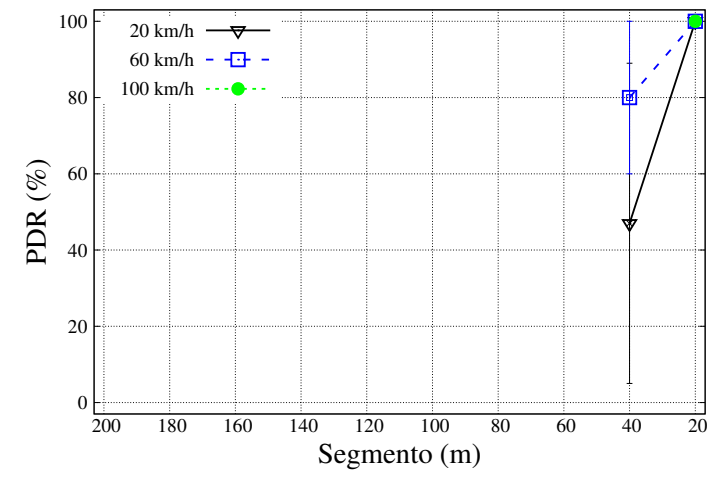

(a) PDR obtida no cenário 3 .

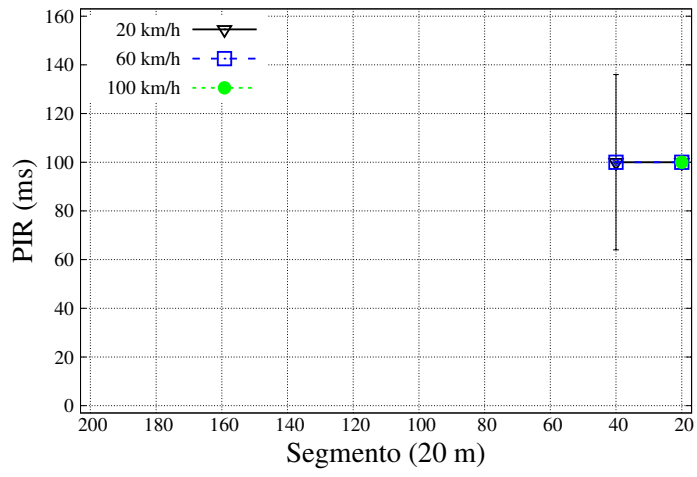

(b) PIR obtido no cenário 3.

Figura 5. Impacto da mobilidade em condições com NLoS.

Dada a atenuação do sinal de rádio devido à condição NLoS, é nítida a diferença da PDR em comparação ao cenário com LoS. Enquanto que no cenário anterior a distância máxima na qual o pedestre conclui o estabelecimento da conexão e inicia a transmissão de BSMs ao veículo é em torno de $140 \mathrm{~m}(20 \mathrm{~km} / \mathrm{h})$, no presente cenário é de aproximadamente $40 \mathrm{~m}$. Isto demonstra que, em condições NLoS, o atraso para estabelecimento da conexão no Wi-Fi Direct pode inviabilizar seu uso em VANETs, já que o tempo para transmissão das BSMs e atuação da aplicação após a recepção pode ser insuficiente. Além disso, no cenário com LoS, a PDR obtida a $20 \mathrm{~km} / \mathrm{h}$ e $40 \mathrm{~m}$ foi $\geq 90 \%$. Já no cenário atual, a PDR neste mesmo segmento foi $<50 \%$. O mesmo padrão de queda é observado nas demais velocidades: se antes era possível obter uma PDR de $100 \%$ a $60 \mathrm{~m}$ e $40 \mathrm{~m}$ trafegando a $60 \mathrm{~km} / \mathrm{h}$ e $100 \mathrm{~km} / \mathrm{h}$, a atenuação do sinal fez com que a PDR nestas mesmas condições fosse $80 \%$ e $100 \%$, porém em $40 \mathrm{~m}$ e $20 \mathrm{~m}$. Dada a distância calculada no cenário com LoS para parar o veículo após a recepção do alerta $(8 \mathrm{~m}, 36 \mathrm{~m}$ e $80 \mathrm{~m})$, há uma grande chance de não ser possível evitar colisões a $60 \mathrm{~km} / \mathrm{h}$ e $100 \mathrm{~km} / \mathrm{h}$ em condições NLoS.

No que se refere ao PIR, diferente do cenário com LoS, nenhuma correlação com a PDR foi observada. Neste cenário, o PIR foi sempre similar à taxa de BSMs (100 ms), apesar da redução da PDR no segmento compreendido entre $20 \mathrm{~m}$ a $40 \mathrm{~m}$ de distância. Tal resultado confirma a proposição de [Renda et al. 2016], ou seja, de que pode não ser 
possível estimar o PIR a partir da PDR. Isto se deve à distinção no padrão de recepção das BSMs, que pode ser variável (recepções alternadas) ou fixo (recepções contínuas). Em condições NLoS, mesmo que em um dado momento não haja recepções devido à obstrução do sinal, após o transmissor obter uma condição de LoS predominante, as BSMs podem ser recebidas de forma imediata. Isto gera um PIR similar à taxa de BSMs, apesar da PDR ser baixa. Portanto, é possível que blackouts de consciência situacional ocorram em tais condições, e o veículo navegue às cegas pela via por algum tempo.

A Tabela 3 apresenta a PDR e o PIR global. Mais uma vez, a PDR é superior a 90\% e o PIR é similar à taxa de geração de BSMs, demonstrando uma tolerância da camada física do Wi-Fi Direct à interferência intersimbólica, independente da velocidade.

Tabela 3. PDR e PIR global por velocidade (NLoS).

\begin{tabular}{|c|c|c|}
\hline Velocidade (km/h) & PDR (\%) & PIR (ms) \\
\hline 20 & 96,42 & 104,52 \\
\hline 60 & 100 & 100,56 \\
\hline 100 & 100 & 100,55 \\
\hline
\end{tabular}

\section{Conclusões e Trabalhos Futuros}

Este trabalho realizou uma análise do desempenho do Wi-Fi Direct através de medições reais utilizando smartphones comerciais. Por meio da comunicação V2P e baseado em uma situação do mundo real - onde pedestres e veículos podem provocar colisões devido à travessias inapropriadas na via sem a devida autorização semafórica - foram investigados o alcance máximo da comunicação, a taxa de entrega de pacotes e o tempo entre recepções de pacotes. Além disso, o impacto de altas velocidades relativas na comunicação, bem como de transmissões realizadas com e sem linha de visada, também foi analisado.

Os resultados indicam que o alcance máximo real do Wi-Fi Direct (PDR $\geq 80 \%$ ) foi de $120 \mathrm{~m}$, apesar de ainda haver recepção de BSMs a $200 \mathrm{~m}$. Dado o atraso para estabelecimento da conexão, no cenário com mobilidade e LoS só é possível receber BSMs em $140 \mathrm{~m}, 60 \mathrm{~m}$ e $40 \mathrm{~m}$, com o veículo a $20 \mathrm{~km} / \mathrm{h}, 60 \mathrm{~km} / \mathrm{h}$ e $100 \mathrm{~km} / \mathrm{h}$, respectivamente. Apesar disso, apenas a $100 \mathrm{~km} / \mathrm{h}$ há o risco de não evitar a colisão entre veículo e pedestre. Já no cenário NLoS, dada a atenuação do sinal, a distância máxima na qual é possível receber BSMs é de $40 \mathrm{~m}$ a $20 \mathrm{~km} / \mathrm{h}$, com a PDR indo de $90 \%$ a $<50 \%$ comparado ao cenário com LoS. Dada a queda da PDR, garante-se que colisões podem ser evitadas apenas a $20 \mathrm{~km} / \mathrm{h}$. Portanto, em condições NLoS, o Wi-Fi Direct pode não ser viável.

Com relação ao PIR, foi possível observar uma correlação negativa com a PDR em alguns casos, especialmente na avaliação do alcance. Onde a PDR foi próxima de $100 \%$, o PIR foi similar à taxa de geração de BSMs $(100 \mathrm{~ms})$, indicando que o Wi-Fi Direct atende ao requisito de frequência de recepção de aplicações de segurança. Nenhum blackout foi registrado durante os experimentos. Por fim, mesmo com o emprego de altas velocidades relativas, os resultados da PDR e do PIR global sugerem uma tolerância do Wi-Fi Direct a interferência intersimbólica gerada pelo deslocamento Doppler.

Como trabalhos futuros, pretende-se propor um mecanismo para envio proativo de BSMs, que não necessite de quadros de requisição. O objetivo é propor um modelo baseado no modo OCB (Outside the Context of a BSS) do IEEE 802.11p, eliminando o 
atraso para formação de grupo do Wi-Fi Direct e permitindo uma melhor atuação em condições NLoS. Pretende-se também explorar a rede embarcada CAN (Controller Area Network) do veículo como forma de integrar a técnica de dead reckoning ao GPS em um modelo para melhoria da precisão da posição geográfica do veículo. Por fim, uma das metas futuras é propor um módulo de comunicação compatível com o Wi-Fi Direct para o NS-3, permitindo avaliações em larga escala.

\section{Agradecimentos}

O presente trabalho foi realizado com apoio da Coordenação de Aperfeiçoamento de Pessoal de Nível Superior Brasil (CAPES) - Código de Financiamento 001. Também foi parcialmente financiado pelo CNPq, FAPERJ e pelo processo ${ }^{\circ}$ 15/24494-8 da Fundação de Amparo à Pesquisa do Estado de São Paulo (FAPESP).

\section{Referências}

Bai, F., Stancil, D. D., and Krishnan, H. (2010). Toward Understanding Characteristics of Dedicated Short Range Communications (DSRC) from a Perspective of Vehicular Network Engineers. In Proceedings of the sixteenth annual international conference on Mobile computing and networking, pages 329-340.

Balasundram, A., Samarasinghe, T., and Dias, D. (2016). Performance Analysis of Wi-Fi Direct for Vehicular Ad-hoc Networks. In 2016 IEEE International Conference on Advanced Networks and Telecommunications Systems (ANTS), pages 1-6. IEEE.

Camps-Mur, D., Garcia-Saavedra, A., and Serrano, P. (2013). Device-to-Device Communications with Wi-Fi Direct: Overview and Experimentation. IEEE wireless communications, 20(3):96-104.

Chaki, P., Yasuda, M., and Fujita, N. (2015). Seamless Group Reformation in WiFi Peer to Peer Network using Dormant Backend Links. In 2015 12th Annual IEEE Consumer Communications and Networking Conference (CCNC), pages 773-778. IEEE.

Consortium, C. V. S. C. et al. (2005). Vehicle Safety Communications Project: Task 3 Final Report: Identify Intelligent Vehicle Safety Applications Enabled by DSRC. National Highway Traffic Safety Administration, US Department of Transportation, Washington DC.

Frank, R., Bronzi, W., Castignani, G., and Engel, T. (2014). Bluetooth Low Energy: An Alternative Technology for VANET Applications. In 2014 11th annual conference on wireless on-demand network systems and services (WONS), pages 104-107. IEEE.

Jeong, S., Baek, Y., and Son, S. H. (2016). A Hybrid V2X System for Safety-Critical Applications in VANET. In 2016 IEEE 4th International Conference on Cyber-Physical Systems, Networks, and Applications (CPSNA), pages 13-18. IEEE.

Jeong, S., Baek, Y., and Son, S. H. (2019). Hierarchical Network Architecture for NonSafety Applications in Urban Vehicular Ad-Hoc Networks. Sensors, 19(19):4306.

Khan, M. A., Cherif, W., Filali, F., and Hamila, R. (2017). Wi-Fi Direct ResearchCurrent Status and Future Perspectives. Journal of Network and Computer Applications, 93:245-258.

Manamperi, W., Samarasinghe, T., and Dias, D. (2018). Enhancing the Wi-Fi Direct Protocol for Data Communication in Vehicular Ad-hoc Networks. In 201821 st International Conference on Intelligent Transportation Systems (ITSC), pages 812-817.

Miucic, R. and Bai, S. (2011). Performance of Aftermarket (DSRC) Antennas Inside a Passenger Vehicle. SAE Int. J. Passeng. Cars-Electron. Electr. Syst., 4:150-155.

Park, Y., Ha, J., Kuk, S., Kim, H., Liang, C.-J. M., and Ko, J. (2014). A Feasibility Study and Development Framework Design for Realizing Smartphone-based Vehicular Networking Systems. IEEE transactions on mobile computing, 13(11):2431-2444. 
Renda, M. E., Resta, G., Santi, P., Martelli, F., and Franchini, A. (2016). IEEE 802.11 p VANets: Experimental Evaluation of Packet Inter-Reception Time. Computer Communications, 75:26-38.

Shahin, A. A. and Younis, M. (2015). Alert Dissemination Protocol using Service Discovery in Wi-Fi Direct. In 2015 IEEE International Conference on Communications $(I C C)$, pages 7018-7023. IEEE.

Statista (2019). Global Smartphone Penetration Rate as Share of Population from 2016 to 2020 .

Su, K.-C., Wu, H.-M., Chang, W.-L., and Chou, Y.-H. (2012). Vehicle-to-Cehicle Communication System through Wi-Fi Network using Android Smartphone. In 2012 International conference on connected vehicles and expo (ICCVE), pages 191-196. IEEE.

Sun, W., Yang, C., Jin, S., and Choi, S. (2016). Listen Channel Randomization for Faster Wi-Fi Direct Device Discovery. In IEEE INFOCOM 2016-The 35th Annual IEEE International Conference on Computer Communications, pages 1-9. IEEE.

Touati, F., Tabish, R., and Mnaouer, A. B. (2013). A Real-Time BLE Enabled ECG System for Remote Monitoring. APCBEE procedia, 7:124-131.

Won, M., Shrestha, A., and Eun, Y. (2018). Enabling WiFi P2P-based Pedestrian Safety App. arXiv preprint arXiv:1805.00442.

Wong, J. Y. (2008). Theory of Ground Vehicles. John Wiley \& Sons.

World Health Organization (2019). Road Traffic Injuries.

Zhang, H., Wang, Y., and Tan, C. C. (2014). WD2: An Improved WiFi-Direct Group Formation Protocol. In Proceedings of the 9th ACM MobiCom workshop on Challenged networks, pages 55-60. ACM. 